$$
34^{316} \quad \cos 2271-14
$$

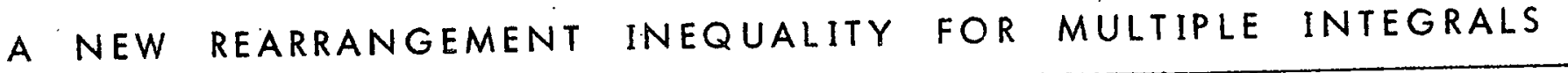

\section{J. M. Luttinger}

Department of Physics

Columbia University, New York, N. Y. 10027

and

\section{R. Friedberg}

Department of Physics :

Columbia University and Barnard College, New York, N. Y. 10027

In this paper we state and prove an inequality (see Eq. (8) below)

for integrals on $n$ variables, of which the case $n=2$ is due to Hardy and Littlewood. In appendices we sketch an alternate proof, as well as a proof of an analogous inequality on discrete sums, and conjecture a further generalization.
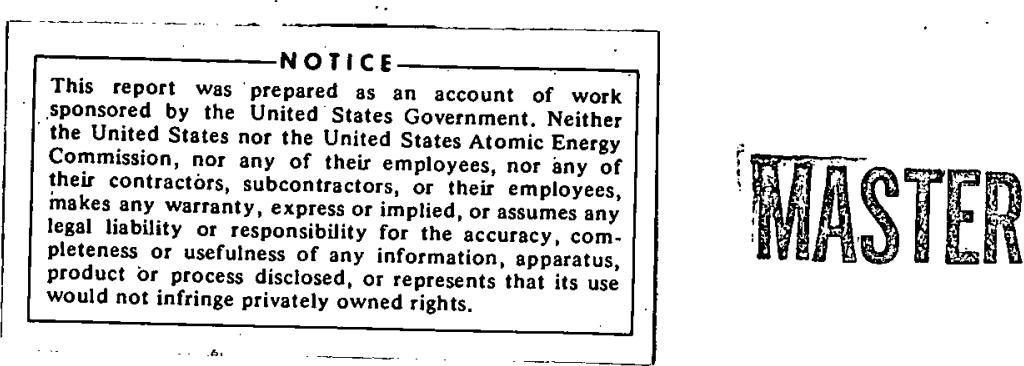

This research was supported in part by the National Science Foundation and by the United States Atomic Energy Commission. 


\section{DISCLAIMER}

This report was prepared as an account of work sponsored by an agency of the United States Government. Neither the United States Government nor any agency Thereof, nor any of their employees, makes any warranty, express or implied, or assumes any legal liability or responsibility for the accuracy, completeness, or usefulness of any information, apparatus, product, or process disclosed, or represents that its use would not infringe privately owned rights. Reference herein to any specific commercial product, process, or service by trade name, trademark, manufacturer, or otherwise does not necessarily constitute or imply its endorsement, recommendation, or favoring by the United States Government or any agency thereof. The views and opinions of authors expressed herein do not necessarily state or reflect those of the United States Government or any agency thereof. 


\section{DISCLAIMER}

Portions of this document may be illegible in electronic image products. Images are produced from the best available original document. 
The subject of rearrangement inequalities is discussed by Hardy, Pólya and Littlewood. 'Briefly, $f$ and $g$ are equimeasurable functions from the interval. $(-\infty, \infty)$ to the interval $[\cdots, \infty)$ if the two sets $\{x \mid f(x) \geq y\}$ and $\{x \mid g(x) \geq y\}$ are equal in measure for each $y$; and the symmetric decreasing rearrangement of $f$ is defined to be that function $f *$ which is equimeasurable with $f$ and satisfies $f *(x)=$ $f *(-x) \geqslant f^{*}\left(x^{\prime}\right)$ when $|x| \leqslant\left|x^{\prime}\right| \because$ This definition leads to various integral inequalities, of which the simplest is

$$
\int F_{1}(x) F_{2}(x) d x \leq \int F_{1}^{*}(x) F_{2}^{*}(x) d x
$$

(all integrals run from $-\infty$ to $\infty$ unless otherwise labeled). The proof of (1) is fairly trivial; see sec. 10.13 of [1]. One can easily generalize it to the product of $n$ functions. Another generalization is

$$
\int F_{1}\left(x_{1}\right) H^{*}\left(x_{1}-x_{2}\right) F_{2}\left(x_{2}\right) d x_{1} d x_{2} \leq \int F_{1}^{*}\left(x_{1}\right) H^{*}\left(x_{1}-x_{2}\right) F_{2}^{*}\left(x_{2}\right) d x_{1} d x_{2}
$$

where the same symmetric decreasing function $H^{*}$ appears on both sides. The inequality (2) reduces to (1) if $\mathrm{H}^{*}$ is taken to be the Dirac $\delta$-function (i.e. in the limit as $H^{*}$ becomes sharply peaked at the origin and vanishes elsewhere).

An easy proof of (2) follows the lines of $\mathrm{sec} .10 .8$ of [1]. This proof can be generalized to the inequality

$$
\begin{aligned}
& \int F_{1}\left(x_{1}\right) H_{1}^{*}\left(x_{1}-x_{2}\right) \ldots F_{n}\left(x_{n}\right) H_{n}^{*}\left(x_{n}-x_{1}\right) d x_{1} \ldots d x_{n} \\
& \quad \leqslant \int F_{1}^{*}\left(x_{1}\right) H_{1}^{*}\left(x_{1}-x_{2}\right) \ldots F_{n}^{*}\left(x_{n}\right) H_{n}^{*}\left(x_{n}-x_{1}\right) d x_{1} \ldots d x_{n}
\end{aligned}
$$

as we shall show in a subsequent paper, in which (3) will be further generalized to functions on a circular domain. 
3.

Henceforth, we shall adopt the convention that

$$
x_{n+1}=x_{1}, x_{0} \equiv x_{n}, d_{0} \equiv H_{n}, \text { atc. }
$$

so that the left side of (3), for example, can be written more compactly either as $\int \prod_{1}^{n}\left[F_{i}\left(x_{i}\right) H_{i}^{*}\left(x_{i}-x_{i+1}\right) d x_{i}\right]$ or as $\int \prod_{1}^{n}\left[H_{i-1}^{*}\left(x_{i-1}-x_{i}\right) F_{i}\left(x_{i}\right) d x_{i}\right]$.

A much more difficult theorem than (2) is obtained by replacing $H^{*}$ on the left by an arbitrary function $H$ of which $H^{*}$ is the symmetric decreasing rearrangement:

$$
\int F_{1}\left(x_{1}\right) H\left(x_{1}-x_{2}\right) F_{2}\left(x_{2}\right) d x_{1} d x_{2} \leq \int F_{1}^{*}\left(x_{1}\right) H^{*}\left(x_{1}-x_{2}\right) F_{2}^{*}\left(x_{2}\right) d x_{1} d x_{2} .
$$

This inequality is proved in sec. $10.14-5$ of [1], in the equivalent form

$$
\int H_{1}\left(y_{1}\right) H_{2}\left(y_{2}\right) H_{3}\left(-y_{1}-y_{2}\right) d y_{1} d y_{2} \leq \int H_{1}^{*}\left(y_{1}\right) H_{2}^{*}\left(y_{2}\right) H_{3}^{*}\left(-y_{1}-y_{2}\right) d y_{1} d y_{2} .
$$

As indicated in sec. 10.9 of [1]; the proof of $(6)$ is easily extended to the generalization

$$
\int H_{n}\left(-\sum_{i}^{n-1} y_{i}\right)_{1}^{n-1}\left[H_{i}\left(y_{i}\right) d y_{i}\right] \leq \int H_{n}^{*}\left(-\sum_{i}^{n-1} y_{i}\right) \prod_{1}^{n-1}\left[H_{i}^{*}\left(y_{i}\right) d y_{i}\right] .
$$

Our purpose in this paper is to prove a much more powerful and more difficult generalization of (5), namely

$$
\int \prod_{i}^{n}\left[F_{i}\left(x_{i}\right) H_{i}\left(x_{i}-x_{i+1}\right) d x_{i}\right] \leqslant \int \prod_{1}^{n}\left[F_{i}^{*}\left(x_{i}\right) H_{i}^{*}\left(x_{i}-x_{i+1}\right) d x_{i}\right]
$$


which includes both (3) and (7) as special cases. (To obtain (7) from (8), replace $F_{2}, \ldots, F_{n}$ by constants and let $y_{i} \equiv x_{i}-x_{i+1}$ for $\left.1 \leqslant i \leq n.\right)$ Both (3) and the more general (8) have recently found application in physics, where they give rise to a class of Green's-function inequalities [2] of which certain well-known isoperimetric inequalities are special cases.

To prove ( 8 , we first observe that it suffices to prove it in the case that each function $F$ or $H$ takes only the values 0 or 1 ; moreover, we may assume that $\{x \mid f(x)=1\} \quad$ is compact and piecewise continuous (the union of a finite number of closed intervals) where $f$ is any $F_{i}$ or $\mathrm{H}_{i}$. (See the discussion in sec. 10.14 of [1].) Therefore (8) is a corollary of the following theorem.

Theorem I.

Let $F_{i}(1 \leq i \leq n)$ be the wharacteristic unction of a compact, piecewise continuous set of measure $m$. on the real Iine and let $F_{i} *$ be the characteristic function of $s_{m_{i}}$ where, for any $m$, we define

$$
S_{m} \equiv\{x|| x \mid \leq m / 2\} \text {. }
$$

Iikewise let $\mathrm{H}_{i}(1 \leq i \leq n)$ be the characteristic function of a set of measure $k_{i}$, and $\mathrm{H}_{i}$ * that of $s_{k_{i}}$.

Then

$$
\int \prod_{i}^{n}\left[F_{i}\left(x_{i}\right) H_{i}\left(x_{i}-x_{i+1}\right) d x_{i}\right] \leqslant \int \prod_{1}^{n}\left[F_{i}^{*}\left(x_{i}\right) H_{i}^{*}\left(x_{i}-x_{i+1}\right) d x_{i}\right]
$$


where $x_{n+1}$ is to be read as an alternate notation for $x_{1}$.

Proof:

We define an operation of truncation as follows:

if $0 \leqslant a_{i} \leq m_{i}$, we let

$$
\begin{aligned}
F_{i}^{a_{i}}\left(x_{i}\right) & =1 \text { if } F_{i}\left(x_{i}=1\right) \text { and } \int_{x_{i}}^{\infty} F_{i}(x) d x \geqslant a_{i} \\
& =0 \text { otherwise; }
\end{aligned}
$$

and if $0 \leqslant a_{i}, 0 \leqslant a_{i+1}, a_{i}+a_{i+1} \leq k_{i}$, we let

$$
\begin{aligned}
H_{i}^{a_{i} a_{i+1}}\left(x_{i}-x_{i+1}\right)=1 \text { if } H_{i}\left(x_{i}-x_{i+1}\right)=1 & \text { and } \int_{x_{i}-x_{i+1}}^{\infty} H_{i}(y) d y \geqslant a_{i} \\
& \text { and } \int_{-\infty}^{x_{i}-x_{i+1}} H_{i}(y) d y \geqslant a_{i+1}
\end{aligned}
$$

$=0$ otherwise.

The truncated function $F_{i}{ }^{a_{i}}$ is the characteristic function of a set obtained from the set represented by $F_{i}$ through the deletion of an upper portion of measure $a_{i}$. Likewise $H_{i}{ }^{a_{L}} a_{i+1}$ represents a set obtained by deleting a measure $a_{i}$ from the top and $a_{i+1}$ from the bottom of the set corresponding to $\mathrm{H}_{i} \cdot($ See $\mathrm{Fig}$. 1.)

We may similarly define : $\quad F_{i}^{* a_{i}} \quad H_{i}^{* a_{i} a_{l+1} \text { by }}$ truncating $\mathrm{F}_{i}{ }^{*}, \mathrm{H}_{i}{ }^{*}$. Alternatively we may define $\mathrm{F}_{i}^{a_{i}}$, 
$\mathrm{H}_{i}^{a_{i} a_{i+1}}$ *as characteristic functions of $\mathrm{S}_{m_{i}-a_{i}}$, $s_{k_{i}-a_{i}-a_{i+1}}$. We note, however, that

$$
\begin{aligned}
& F_{i}^{* a_{i}}\left(z_{i}\right)=F_{i}^{a_{i}^{\prime \prime}}\left(x_{i}+a_{i}\right)^{\prime} \\
& H_{i}^{* a_{i} a_{i+1}}\left(x_{i}-x_{i+1}\right)=H_{i}^{a_{i} a_{i+1}}\left(x_{i}+\frac{1}{2} a_{i}-x_{i+1}-\frac{1}{2} a_{i+1}\right) \text {. }
\end{aligned}
$$

(See Fig. 2.)

The passage from $F$ to $F^{a}$ is equivalent to the passage from $F$ to $F_{1}$ in sec. 10.15 of [1], except that Hardy et al. arrange to let a have only integer values. The difference is a matter of taste. By making a vary continuously, we approach more closely the method of Riesz (sec.10.16 of [1]) except that he truncates symmetrically by deleting a set of measure $t$ simultaneously from top and bottom. (see footnote, p. 286 of [1].) In this matter we have no choice; it will be essential that in forming the double truncation $\mathrm{H}^{a b}$ we do not allow the parameters $a$ and $b$ to interfere with one another, and this is achieved by letting them act at opposite ends as specified by (12).

To give the reader a clear idea of how our truncation is related to that of Hardy et al., let us paraphrase their proof (secil0.15 of [1]) of theorem $I$ in the case $n=2$. First we drop the factor. $\mathrm{H}_{2}$ from (10) because it is inessential in this case, having the same argument as $H_{1}$. Then we point out that (10) must hold if $m_{1}+k_{1} \leqslant m_{2}$, because the right side then takes the value $m_{1} k_{1}$, and the left side cannot be more. 
Now suppose that $m_{1}+k_{1}>m_{2}$, and let

$$
\begin{aligned}
I(a) & =\int F_{1}^{a}\left(x_{1}\right) H_{1}^{a o}\left(x_{1}-x_{2}\right) F_{2}\left(x_{2}\right) d x_{1} d x_{2}, \\
I(a) & =\int F_{1}^{* a}\left(x_{1}\right) H_{1}^{* a o}\left(x_{1}-x_{2}, F_{2}^{*}\left(x_{2}\right) d x_{1} d x_{2},\right. \\
A & =\min \left(m_{1}, k_{1}, k_{2}\left(m_{1}+k_{1}-m_{2}\right)\right) .
\end{aligned}
$$

We already know that $I(A) \leq{ }^{*} I(A)$-note the use of $(13)$ - and we must prove $I(0) \leq I(0)$. Thus it suffices to show that $-\frac{d I}{d a} \leqslant-\frac{d I}{d a}$ for $0 \leqslant a<A$. This is done by showing on the one hand that

$$
-\frac{d}{d a} \int F_{1}^{a}\left(x_{1}\right) H_{1}^{a}\left(x_{1}-x_{2}\right) d x_{1} \leq 1 \text { for all } x_{2}
$$
(compare Eq: (10.15.16) of [1]) so that $-\frac{d I}{d a} \leq m_{2}$; and on the other hand that

$$
-\frac{d}{d a} \int F_{1}^{* a}\left(x_{1}\right) H_{1}^{* a 0}\left(x_{1}-x_{2}\right) d x_{1}=1 \text { for }\left|x_{2}\right| \leqslant \frac{1}{2}\left(m_{1}-a\right)+y_{2}\left(k_{1}-a\right),
$$

so that $-\frac{d^{*} I}{d a}=m_{2}$ exactly (see Eq. (10.15.13) of [1]). The proof of Riesz (sec. 10-16 of [1]) is essentially the same.

It is possible to extend this proof to $n>2$; see Appendix I. However, the proof we shall presen is based instead on the following variation of the above argument for $\mathrm{n}=2$.

Without loss of generality we may suppose that $m_{1} \geq m_{2}$. If $m_{1}>m_{2}$, we define $I(a), I(a)$ as above, but let $A=\min \left(k_{1} ; m_{1}-m_{2}\right)$. As above, we can prove $-\frac{d I}{d a} \leqslant-\frac{d I}{d a}$ for $0 \leq a<A$, and so it suffices to show that $I(A) \leqslant I(A)$. This is trivial if $A=k_{1}$, and if $A=m_{1}-m_{2}$ it reduces to the original theorem with 
8.

We treat the case $m_{1}=m_{2}$ by defining

$$
\begin{aligned}
I(a, b) & =\int F_{1}^{a}\left(x_{1}\right) H_{1}^{a b}\left(x_{1}-x_{2}\right) F_{2}^{b}\left(x_{2}\right) d x_{1} d x_{2} \\
I(a, b) & =\int F_{1}^{a}\left(x_{1}\right) H_{1}^{* a b}\left(x_{1}-x_{2}\right) F_{2}^{* b}\left(x_{2}\right) d x_{1} d x_{2} \ldots \\
B & =\min \left(m_{2}, 1 / 3 k_{1}\right)
\end{aligned}
$$

Then $I(B, B)=0=I(B, B)$, so that it suffices to prove $-\frac{d}{d b} I(b, b) \leq-\frac{d}{d b}+I(b, b) \quad$ for $0 \propto B \quad$ By a repetition of earlier arguments we find that $-\frac{\partial}{\partial a} I(a, b) \leq-\frac{\partial}{\partial a}+I(a, b) \quad$ if,$a \leq b ;$ and $\quad-\frac{\partial}{\partial b} I(a, b) \leq-\frac{\partial}{\partial b} I(a, b)$ if $b=a$.

Setting $a=b$ and adding, we obtain the desired inequality.

The advantage of this longer argument is that it avoids reduction to the inequality $I(A) \leq I(A)$ for $A=\frac{1}{2}\left(m_{1}+k_{1}-m_{2}\right) \quad-$ the case $\mu=0$ in sec. 10.15 of [1]. In Appendix I we Indicate how this step can be generalized to $n>2$, if one prefers the first argument.

We now return to the proof of Theorem I for arbitrary n. We begin, by defining

$$
\begin{aligned}
I\left(a_{1} \ldots a_{n}\right) & =\int \pi_{1}^{n}\left[F_{i}^{a_{i}}\left(x_{i}\right) H_{i}^{a_{i} a_{i+1}}\left(x_{i}-x_{i+1}\right) d x_{i}\right] \\
I\left(a_{1} \ldots a_{n}\right) & =\int \pi_{1}^{n}\left[F_{i}^{a_{i} *}\left(x_{i}\right) H_{i}^{a_{i} a_{i+1}^{*}}\left(x_{i}-x_{i+1}\right) d x_{i}\right] \\
& =\int \prod_{1}^{n}\left[F_{i}^{* a_{i}}\left(x_{i}\right) H_{L}^{* a_{i} a_{i+1}}\left(x_{i}-x_{i+1}\right) d x_{i}\right]
\end{aligned}
$$


the two lines of (15) being equivalent because of (13).

Let $0 \leq j<n$. We shall obtain (10) as a special case $(j=0)$ of the following theorem.

Theorem IA.

Suppose that

and

$K_{i}>0$, for $i=1, \ldots, j$

Then $m_{n} \leqslant m_{i}$, for $i=1, \ldots, n-1$.

$$
\prod_{1}^{j}\left(-\frac{\partial}{\partial a_{i}}\right) I\left(a_{1} \ldots a_{n}\right)_{0}=\prod_{1}^{j}\left(-\frac{\partial}{\partial a_{i}}\right) I\left(a_{1} \ldots a_{n}\right)_{0}(18)
$$

where the final subscript 0 means that all the $a_{i}$ are set equal to 0 after differentiation.

The proof of Theorem IA will proceed by double induction. Let $n=2+j$ : We shall prove, first, that Theorem IA holds for $l=l$ and arbitrary $j$; and second, that if it holds for $\ell-1$ (and any $j$ ) then it holds for $\mathcal{L}$ (and any $j$ ). In the latter part, we shall use induction on $j$ for fixed $\ell$, starting with $j=0$.

After proving Theorem IA, we shall deriva Theorem I from it. So as to make the logical structure of the proof readily visible, we shall defer the demonstration of certain statements to notes following the main argument.

The case $l \cdot l$ is treated by showing (Note l) that the function

$$
\begin{aligned}
h\left(x_{n}\right)= & \prod_{1}^{n-1}\left(-\frac{\partial}{\partial a_{i}}\right)\left\{\int H_{n}^{a_{n} a_{i}}\left(x_{n}-x_{1}\right)\right. \\
& \left.\prod_{1}^{n-1}\left[F_{i}^{a_{i}}\left(x_{i}\right) H_{i}^{a_{i} a_{i+1}}\left(x_{i}-x_{i+1}\right) d x_{i}\right]\right\}_{0}
\end{aligned}
$$


10.

takes on only 0 and $I$ as values, whereas (Note 2) the corresponding function

$$
\begin{aligned}
h\left(x_{n}\right) & =\prod_{1}^{n-1}\left(-\frac{\partial}{\partial a_{i}}\right)\left\{\int H_{n}^{* a_{n} a_{i}}\left(x_{n}-x_{1}\right)\right. \\
& \left.\prod_{1}^{n-1}\left[F_{i}^{a_{i}}\left(x_{i}\right) H_{i}^{\left(a_{i} a_{l}+1\right.}\left(x_{i}-x_{i+1}\right) d x_{i}\right]\right\}_{0}
\end{aligned}
$$

is the characteristic function of $S_{\bar{m}}$ where

$$
\dot{\bar{m}} \geq \min \left\{m_{i}\right\}
$$

Writing (18) in the form

$$
\int F_{n}\left(x_{n}\right) h_{n}\left(x_{n}\right) d x_{n} \leqslant \int F_{n}^{*}\left(x_{n}\right) h_{n}\left(x_{n}\right) d x_{n} \quad \text { (22) }
$$

we see from Note 1 that the left side cannot exceed $\int F_{n}\left(x_{n}\right) d x_{n}=m_{n}, \quad$ and from Note 2 that the right side is min $\left(m, m_{n}\right)$ which by $(21)$ and $(17)$ is $m_{n}$. This verifies (22), and hence (18) when $l=l$.

Now suppose that $l \otimes 1$ and that Theorem IA holds when $\mathcal{L}$ is reduced by 1 .

Define

$$
\begin{aligned}
& g(a)=\prod_{1}^{j}\left(-\frac{\partial}{\partial a_{i}}\right) I\left(a_{1} \ldots a_{n}\right)_{0, a} \\
& g(a)=\prod_{1}^{j}\left(-\frac{\partial}{\partial a_{i}}\right) I\left(a_{1} \ldots a_{n}\right)_{0, a}
\end{aligned}
$$

where the final subscripts mean that we set $a j+1=a$ and all other $a_{i}=0$ after differentiation. Then (18) may be written

$g(0) \leq g^{\prime}(0) \quad(25)$ 
Define

$$
A=\min \left(k_{j+1}, k_{j}, m_{j+1}-m_{n}\right) \text {. }
$$

(We read $k_{0}$ as $k_{n}$ according to (4).) Then for any a satisfying $0 \leq a<A$, we see that, (16) and (17) are still satisfied if $m_{j+1}$, $k_{j}, k_{j+1}$ are al1 diminished by $a$.

It follows that

$$
-\frac{d}{d a} g(a)=-\frac{d}{d a} * g(a)
$$

for all $0 \leqslant a<A$, as an application of Theorem IA with $l \rightarrow l+1 \quad$ - Therefore, to prove (25) it. suffices to prove

$$
g(A) \leqslant{ }^{*} g(A)
$$

We distinguish three cases.

Case 1: $A=k_{j+1}$, or $j=0$ and $A=k_{0}\left(j k_{n}\right)$. Here (28) is obviously true since both sides vanish.

Case 2: $j>0$ and $A=k_{j}<k_{j+1}$. This is really the most difficult case. Although the integrand in $g(a)$ contains the factor $\mathrm{H}_{j} a^{a}$ which vanishes for $a_{j}=0$ and $a=A, g(A)$ itself does not vanish because the operator $-\frac{\partial}{\partial a_{j}}$ is to be applied before letting $a \rightarrow A-$. However, we show in Note 3 that (28) may be transformed, in this case, into an application of Theorem IA with $l \rightarrow l$ and $j \rightarrow j-1$, by eliminating the variable $x_{j}$. Thus eventually we arrive at $j=0$, when Case 2 can no longer occur.

Case 3: $A=m_{j+1}-m_{n}\left\langle\min \left(k_{j}, k_{j+1}\right)\right.$. Here (28) may be regarded as an example of (25) with $m_{j+1}, k_{j}, k_{j+1}$ al1 diminished by $A$. Conditions (16) and (17) remain 
satisfied, but there is now the additional equality

$$
m_{j+1}=m_{n} \text {. }
$$

It therefore suffices, in order to complete the proof of (25), to prove it in the presence of (29).

We cannot proceed how according to (23) and (24), because if we truncate any further with respect to the variable $x_{j+1}$ (thus diminishing $\mathrm{m}_{j+1}$ ) we shall destroy the validity of (17), which is needed in proving the case $l=1$ on which our induction is based. Instead, we truncate simultaneously with respect to $x_{j+1}$ and $x_{n}$, maintaining (29), and hence (17), throughout. (since $l>1, \dot{x}_{j+1}$ and $x_{n}$ must be distinct.) Thus we shall reduce (25) to a statement analogous to (28) which will be proved true in every case. Assuming (29), we define

$$
\begin{aligned}
& g(a, b)=\prod_{j}^{j}\left(-\frac{\partial}{\partial a_{i}}\right) I\left(a, \ldots a_{n}\right)_{0, a, b} \\
& { }_{g} g(a, b)=\prod_{1}^{j}\left(-\frac{\partial}{\partial a_{i}}\right) I\left(a_{1} \ldots a_{n}\right)_{0,0, b}
\end{aligned}
$$

where we are to set $a_{j+1}=a, a_{n}=b$, all other $a_{i}=0$ after differentiation: our real intention is to put $a=b$, but we keep the two parameters distinct on order to define partial derivatives. We may replace $(25)$, then, by

$$
g(0,0) \leqslant g(0,0)
$$


Let us assume for the moment that $j>0$ and $l>2$. We define

$$
B=\min \left(k_{j+1}, k_{n+1}, k_{j}, k_{n}\right) \text {, }
$$

and note that if $O \leq a<b<B$, conditions (16) and (17) will still hold if $m_{j+1}, k_{j}, k_{j+1}$ are all diminished by a and $m_{n}$, $k_{n}, k_{n-1}$ are al1 diminished by $b$. Using the notation

$g_{1}=\partial g / \partial a, g_{2}=\partial g / \partial b \quad$, and similarly for ${ }^{*} g$, we obtain

$$
-g_{1}(a, b) \leqslant-g_{1}^{*}(a, b)
$$

for $0 \leqslant a \leqslant b<B$ by induction on $l$ in analogy with (27). By a renumbering (Note 4) of the indices $1 . . n$, we also obtain

$$
-g_{2}(a, b) \leqslant-g_{2}(a, b)
$$

for $O \leqslant b \leqslant a<B$ and the sum of (34) and (35) for $a=b$ givees

$$
-\frac{d}{d b} g(b, b) \leqslant-\frac{d}{d b} * g(b, b)
$$

for $0 \leq b<B$. To prove (32), therefore, it suffices to prove

$$
g(B, B) \leqslant * g(B, B)
$$

We distinguish three cases of (37), analogous to those of (28).

$$
\text { Case I: } B=k_{j+1} \text { or } k_{n+1} \text { : Then } g(B, B)=0={ }_{g}(B, B) \text {. }
$$


Case 2a: $\quad B=k_{j} \leqslant \min \left(k_{j+1}, k_{n-1}\right)$. This is handled by induction on $j$ according to Note 3 .

Case $2 b: \quad B=k_{n}<\min \left(k_{j+1}, k_{n-1}\right)$. This is reduced to case $2 a$ by renumbering the indices according to Note 4.

$$
\text { Case 3: } B=m_{n} \text {. Here } g(B, B)=0={ }^{*} g(B, B) \text { as }
$$
in Case 1.

If lal or $j=0$, the proof of (32) given above must be modified slightly (Note 5).

The proof of Theorem IA is now complete. To obtain Theorem I, we cyclically permute the indices $1 \ldots$ n so as to satisfy (17). If (16) holds, we can apply Theorem IA to obtain (18), which for $j=0$ reduces to (10). If (16) fails, (10) follows anyway since both sides vanish. But (10) is invariant under the cyclic permutation of indices; therefore it still holds under the original numbering. This proves Theorem I.

Note 1. We study the right side of (19) by means of the following lemma, which we state in two parts.

Lemma IA. Let $i<n$, and let $g\left(x_{i}\right)$ be the characteristic function of some compact, piecewise continuous set of measure $p$. Let $g^{a l}\left(x_{i}\right)$ be the truncation of $g$ according to (11), for $0 \leqslant a_{i}<p$. Define

$$
G\left(x_{i+1}\right)=-\frac{d}{d a_{i}}\left[\int g^{a_{i}}\left(x_{i}\right) F_{i}^{a_{i}}\left(x_{i}\right) H_{i}^{a_{i} 0}\left(x_{i}-x_{i+1}\right) d x_{i}\right]_{0}
$$

where the final subscript means that we set $a_{i}=0$ after differentiation. Then $G\left(x_{i+1}\right)$ takes only the values 0 and 1 . 
Lemma IB. Moreover, let $G^{a}\left(x_{i+1}\right)$ be the truncation of $G$ according to (11), and let ${ }^{a} G\left(x_{i+1}\right)$ be defined by

$$
{ }^{a} G\left(x_{i+1}\right)=-\frac{d}{d a_{i}}\left[\int g^{a_{i}}\left(x_{i}\right) F_{i}^{a_{i}}\left(x_{i}\right) H^{a_{i} a_{i+1}}\left(x_{i}-x_{i+1}\right) d x_{i}\right]_{0, a}
$$

where we set $a_{i}=0, a_{i+1}=a$. Then either (Case 1)

${ }^{a} G\left(x_{i+1}\right)=G\left(x_{i+1}\right)$ for all $x_{i+1}$ and all a from 0 to $k_{i}$,

or (Case 2) ${ }^{a} G\left(x_{i+1}\right)=G^{a}\left(x_{i+1}\right)$ for all $x_{i+1}$

and all a from 0 to $k_{i}$.

To prove Lemma IA, let $q_{1}, q_{2}, q_{3}$ be the highest numbers satisfying $g\left(q_{1}\right)=F_{i}\left(q_{2}\right)=H_{i}\left(q_{3}\right)=1$. Let $q=q\left(x_{i+1}\right)=\min \left(q_{1}, q_{2}, x_{i+1}+q_{3}\right)=\min \left(q^{\prime}, x_{i+1}+q_{3}\right)$ where $q^{\prime}=\min \left(q_{1}, q_{2}\right)$. Then for any particular $x_{k+1}$, the set $\left\{x_{i} \mid g\left(x_{i}\right) F_{i}\left(x_{i}\right) H_{i}\left(x_{i}-x_{i+1}\right)=1\right\}$

has as highest member a number $r \leqslant q$. The effect on this set of infinitesimal truncation performed simultaneously on 9, $F_{i}, H_{i}$ is to lower $q$ and thereby delete a single infinitesimal portion of the set, if $r=q$; or to leave the set unchanged, if $r<q$. In the former event $G\left(x_{i+1}\right)=1$; in the latter event, $G\left(x_{i+1}\right)=0$. To prove Lemma IB, we observe that

$$
\begin{aligned}
\left\{x_{i+1} \mid G\left(x_{i+1}\right)=1\right\} & =\left\{x_{i+1} \mid g(q) F_{i}(q) H_{i}\left(q-x_{i+1}\right)=1\right\} \\
& =T_{1} \cup T_{2}
\end{aligned}
$$


where

$$
\begin{aligned}
& T_{1}=\left\{x_{i+1} \mid H_{i}\left(q^{\prime}-x_{i+1}\right)=1 \& g\left(q^{\prime}\right) F_{i}\left(q^{\prime}\right)=1\right\} \\
& T_{2}=\left\{x_{i+1} \mid q \leqslant q^{\prime} \& g(q) F_{i}(q)=1\right\}
\end{aligned}
$$

The sets $T_{1}$ and $T_{2}$ are restricted to $x_{i+1} \geqslant q^{\prime}-q_{3}$ and $x_{i+1} \leq q^{\prime}-q_{3} \quad$ respectively, so that they intersect at most at one point. We note also that $q^{\prime}$, and hence $g\left(q^{0}\right) F_{i}\left(q^{0}\right)$ is independent of $x_{i+1}$ Now suppose (Case 1 ) that $g\left(q^{\prime}\right) F_{i}\left(q^{\prime}\right)=0$. (see Fig. 3.) Then $T_{1}$ is empty and from (40) and (42) we find

$$
G\left(x_{i+1}\right)=g\left(x_{i+1}+q_{3}\right) F_{i}\left(x_{i+1}+q_{3}\right)
$$

for all $x_{i \rightarrow 1}$ - But if $H_{i}$ had been truncated from below as in (39), the value of $q_{3}$ would not be altered; i.e., the sets $\left\{y \mid H_{i}^{D_{a}}(y)=1\right\}$ and $\left\{y \mid H_{i}(y)=1\right\}$ have the same highest member. Therefore, in this case, ${ }^{a} G$ is the same function as $G$, for $0 \leqslant a<k_{i}$

suppose instead (Case 2) that $g\left(q^{\prime}\right) F_{i}\left(q^{\prime}\right)=1$. (see Fig. 4.) Then the only way that ${ }^{a_{G}}$ can differ from $G$ is through the replacement of $\mathrm{H}_{i}$ by $\mathrm{H}_{i}^{\mathrm{Oa}}$ in (41). Since $x_{i+1}$ occurs with a negative sign in the argument, the effect of this replacement is just to delete a set of measure 
a from the top of $T_{1}$ - But $T_{1}$ lies "above" $T_{2}$, so that this is the same as truncating $G$ from above. Thus ${ }^{a_{G}}$ and $\mathrm{G}^{a}$ are the same function for $O \leq a<k_{i}$. This completes the proof.

Lemma IA corresponds to the kernel of the Hardy $=L i t t l$ ewood-Riesz proof (see footnote c, p. 284 of [1]). Lemma IB is required for $n>2$, as we shall see in the following argument. For $1 \leq i \leq n$, let us define

$$
h_{i}\left(x_{i}, z\right)=\prod_{1}^{i-1}\left(-\frac{\partial}{\partial a_{s}}\right)\left\{\int H_{n}^{a_{n} a_{1}}\left(z-x_{1}\right) \prod_{1}^{i-1}\left[F_{s}^{a_{s}}\left(x_{s}\right) H_{s}^{a_{s} a_{s+1}}\left(x_{s}-x_{s+1}\right) d x_{s}\right]\right\}_{0}
$$

with the conventions of (4) and (18). We note that $h_{n}\left(x_{n}, x_{n}\right)$ is identical to $h\left(x_{n}\right)$ as defined by (19). Our aim is to prove that this function takes only the values 0 and 1 . To this end, we assert that for each $i<n, h_{i}\left(x_{i}, z\right)$ takes only the values 0 and 1 . Moreover, if $h_{i}^{i}$ is the truncation of $h_{i}$ according to (II), regarded as a function of $x_{i}$ with fixed $z$, and $h_{i}$ is the result of setting $a_{i}=a$ instead of 0 in (44), then ${ }^{a} h_{i}$ is identical, for fixed $z$, either to $h_{i}$ or to $h_{i}^{a} s$, the choice depending on $z$ but not on $a$. We prove these statements by induction on $i$. Since $h_{1}\left(x_{1}, z\right)=H_{n}\left(z-x_{1}\right), a_{h_{1}}\left(x_{1}, z\right)=H_{n}^{o a}\left(z-x_{1}\right)$, the proof is immediate for $i=1$. Now suppose the assertions true of $h_{i}, i<n-1$; we must prove them for $h_{i+1}$. From (44) we obtain 
$h_{i+1}\left(x_{i+1}, z\right)=-\frac{\partial}{\partial a_{i}}\left[\int^{a_{i}} h_{i}\left(x_{i}, z\right) F_{i}^{a_{i}}\left(x_{i}\right) H_{i}^{a_{i} \Delta}\left(x_{i}-x_{i+1}\right) d x_{i}\right]_{0}$

and

$$
{ }^{a} h_{i+1}\left(x_{i+1}, z\right)=-\frac{\partial}{\partial a_{i}}\left[\int^{a_{i}} h_{i}\left(x_{i}, z\right) F_{i}^{a_{i}}\left(x_{i}\right) H_{i}^{a_{i} a_{i+1}}\left(x_{i}-x_{i+1}\right) d x_{i}\right]_{0, a}
$$

with the convention of (39). If $z$ is such that $a_{i} h_{i}$ and $h_{i}^{a_{i}}$ are identical, then these two equations are identical to (38) and (39) when we set $g=h_{i}, G=h_{i+1}$ and suppress the argument $z$. If $z$ is such that ${ }^{a_{i}} h_{i}$ and $h_{i}$ are identical, we can still recover (38) and (39) by setting $G=h_{i+1}$ and defining $g\left(x_{i}\right)=1$ if $h_{i}\left(x_{i}, z\right)=1$ or $q_{2} \leq x_{i} \leq q_{2}+k_{i}$, where $q_{2}$ is the highest number such that $F_{i}\left(q_{2}\right)=1$. For then we have $g^{a_{i}} F_{i}^{a_{i}}=g F_{i}^{a_{i}}=h_{i} F_{i}^{a_{i}}=a_{i} h_{i} F_{i} a_{i}$ for $0=a_{i}<k_{i}$. In either case, the desired properties of $h_{i+1}$ follow from Lemma $I$.

$$
\text { Now taking } i=n-1 \text {, we apply only Lemma IA to }
$$
show from (45) that $h_{i+1}$ takes the values 0 and 1 alone. This is the desired result needed to treat (19).

We may observe that the value of $z$ affects the form of the successive $h_{i}\left(x_{i}, z\right)$ in a complicated way through its effect on the choices between Case 1 and case 2 in the successive applications of Lemma IB. Therefore, it is very difficult to say anything about the $\operatorname{set}\left\{x_{n} \mid h_{n}\left(x_{n}, x_{n}\right)=1\right\}$. This point is what makes (8) so much harder to prove than (7), and it is for this that the condition (17) is vital. 
19.

Note 2. We study the right side of (20) by means of the following lemma.

Lemma IIA - Let $g_{1}^{*}, g_{2}^{*}$ be the characteristic functions of $S_{p_{1}}, S_{p_{2}}$ as defined by $(9)$, and let $g_{1}^{*^{a}}, g_{2}^{*^{a}}$ be their truncations for 0 a $0 \leqslant a<\min \left(p_{1}, p_{2}\right)$, in analogy with (11). Then if $1 \leqslant i<n$, the function

$$
G\left(x_{i+1}, x_{n}\right)=-\frac{d}{d a_{i}}\left[\int g_{1}^{* a_{i}}\left(x_{i}-x_{n}\right) g_{2}^{* a_{i}}\left(x_{1}\right) H_{i}^{* a_{i} \theta}\left(x_{i}-x_{i+1}\right) d x_{i}\right]_{0}
$$

can be expressed in the form

$$
G\left(x_{i+1}, x_{n}\right)=f_{12}^{*}\left(x_{n}\right) f_{13}^{*}\left(x_{i+1}-x_{n}\right) f_{23}^{*}\left(x_{i+1}\right)
$$

where $f_{12}^{*}, f_{13}^{*}, f_{23}^{*}$ are the characteristic functions of $S_{p_{1}}+p_{2}$, $S_{p_{1}+k_{i}}, S_{p_{2}+k_{i}}$ respectively.

Lemma IIB. Moreover, if

$$
{ }^{a} G\left(x_{i+1}, x_{n}\right)=-\frac{d}{d a_{i}}\left[\int g_{1} a_{i}\left(x_{i}-x_{n}\right) g_{2}^{a_{i}}\left(x_{i}\right) H_{i}^{a_{i} a_{i+1}\left(x_{i}-x_{i+1}\right) d x_{i}}\right]_{0, a}
$$

with the convention of (39), then

$$
{ }^{a} G\left(x_{i+1}, x_{n}\right)=f_{12}^{*}\left(x_{n}\right) f_{13}^{* a}\left(x_{i+1}-x_{n}\right) f_{23}^{* a}\left(x_{i+1}\right)
$$

where $f_{13}^{* a}, f_{23}^{* a}$ are the truncations of $f_{13}^{*}, f_{23}^{*}$ according to (11).

Proof of lemma. For fixed $x_{i+1}$ and $x_{n}$, we define $q_{1}^{*}=x_{n} \pm k_{2} p_{1}, q_{2}^{ \pm}= \pm y_{2} p_{2}, q_{3}^{ \pm}=x_{i+1} \pm k_{2} k_{i}$. Then the integrand in (47) is the characteristic function of the set $\left\{x_{i}\left(q^{-} \leq x_{i} \leqslant q^{+}\right\}\right.$ where $q^{+}=\min \left(q_{1}^{+}, q_{2}^{+}, q_{3}^{+}\right)$ 
and $q^{-}=\max \left(q_{1}^{-}, q_{2}^{-}, q_{3}^{-}\right)$. The effect of an infinitesimal truncation is to lower $q_{+^{*}}$ It follows that $G\left(x_{i+1}, x_{n}\right)$ takes the value 1 or 0 according as $q^{+} \geqslant q^{-}$ or not.

Now, the condition $q^{+} \geqslant q^{-}$is equivalent to the six conditions $q_{1}^{+} \geqslant q_{2}^{-}, q_{2}^{+} \geqslant q_{1}^{-}, q_{2}^{+} \geqslant q_{3}^{-}, q_{3}^{+} \geqslant q_{2}^{-}, q_{3}^{+} \geqslant q_{1}^{-}, q_{1}^{+} \geqslant q_{3}^{-}$ which are in turn equivalent to the three conditions

$$
\left|x_{n}\right| \leqslant \frac{1}{2}\left(p_{1}+p_{2}\right),\left|x_{i+1}-x_{n}\right| \leqslant \frac{1}{2}\left(p_{1}+k_{i}\right),\left|x_{i+1}\right| \leqslant 1 / 2\left(p_{2}+k_{i}\right) .
$$

Equation (48) follows immediately. This proves Lemma IIA.

Lemma IIB can be reduced, by the substitution

$x_{i+1} \rightarrow x_{i+1}-y_{2} a$ and the use of (13), to an application of Lemma IIA with $k_{i} \rightarrow k_{i}-a$

To treat the right side of $(20)$, let us represent its integrand by a graph containing points $P_{0}, P_{1}, \ldots, P_{n}$ joined by lines of fictitious "length" according to the following rule. To each factor $F_{i} a_{i}$ in (20) there corresponds a line of "length" $m_{i}$ joining $P_{0}$ to $P_{i}$; to each factor

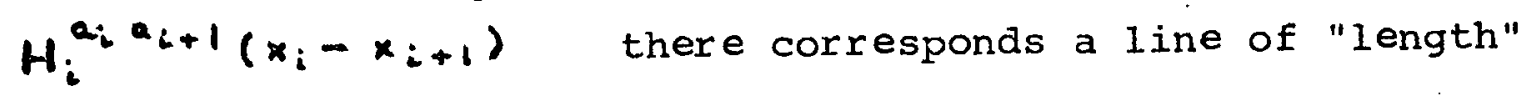
$k_{i}$ joining $P_{i}$ to $P_{i+1}$. (see Fig. 5.)

We now integrate over $x_{1}$, apply the operator $-\frac{\partial}{\partial a_{1}}$, and set $a_{1}=0$. Lemma II tells us that the result corresponds to a new graph obtained by deleting the point $P_{1}$ and its associated lines, and connecting the pairs $\left(P_{n}, P_{0}\right),\left(P_{n}, P_{2}\right),\left(P_{0}, P_{2}\right)$ by new lines of "length" $k_{n}+m_{1}, k_{n}+k_{1}, m_{1}+k_{1}$. It is easily seen that this operation does not change the "length" of the shortest path between any pair of points other than P. 
The new graph has two lines connecting $P_{0}$ to $P_{2}$. They represent two factors $f_{23}^{a_{2}}\left(x_{2}\right)$ and $F_{2}^{a_{2}}\left(x_{2}\right)$, of which the one corresponding to the "Ionger" Iine is superfluous. Therefore, the "longer" Iine can be removed. This operation also leaves unchanged the shortest path between any two points in the graph.

The above process can be repeated so as to eliminate the points $P_{\mathbf{z}}, \ldots, P_{n-1}:$ At the $i^{\text {th }}$ step we integrate over $x_{i}$, operate with $-\frac{\partial}{\partial a_{i}}$ and set $a_{i}=0$. By Lemma. II the graphical result is to delete $P_{i}$ and replace the lines joining $P_{i}$ to its neighbors by new lines joining. each pair of such neighbors with "length" equal to that of the path which formerly linked them indirectly through $P_{i}$. Superfluous lines are then removed.

The end result of the algebraic operations is $* h\left(x_{n}\right)$ as given by (20); that of the graphical operations is a graph consisting of $P_{0}$ and $P_{n}$ joined by a line of some "length" $\bar{m}$. Therefore $*_{h}\left(x_{n}\right)$ is the characteristic function of $s_{\bar{m}}$. This $\bar{m}$ is equal to the "Iength" of the shortest path from $P_{0}$ to $P_{n}$ in the original graph, since this "length" was not altered by any of the graphical changes. But any such path included a step from $P_{0}$ to some $P_{i}$ having "length" $m_{i}$. Consequently (2I) holds. Note 3 .

From the role played by (28) in the proof of (25), it is clear that $g(a)$ and ${ }^{\prime} g(A)$ must be understood as the limits, of $g(a)$ and $* g(a)$ as $a \rightarrow A$ from below. Letting $0 \leq a<A$, we can write $g(a)$, since $\ell>1$ and $j>0$, in the form 
22.

$$
g(a)=\int d x_{j+1} d x_{n} Q\left(a, x_{j+1}, x_{n}\right) a_{h+1}\left(x_{j+1}, x_{n}\right)
$$

where

$$
\begin{aligned}
Q\left(a, x_{j+1}, x_{n}\right) & =\int F_{j+1}^{Q}\left(x_{j+1}\right) H_{j+1}^{a 0}\left(x_{j+1}-x_{j+2}\right) \ldots F_{n}\left(x_{n}\right) d x_{j+2} \cdots d x_{n-1} \\
& \leqslant F_{n}\left(x_{n}\right) m_{j+2} \cdots m_{n-1}
\end{aligned}
$$

and $a_{h_{j+1}}\left(x_{j+1}, x_{n}\right)$ is defined by (44) with $a_{i} \rightarrow a$.

By referring to Note 1 , we see that ${ }^{a} h_{j+1}\left(x_{j+1}, x_{n}\right)$

takes on only the values 0 and 1 , and that, except at one value of $x_{j+1}$, it is the sum of two terms. The first term (corresponding to $T_{1}$ in the proof of Lemma $I B$ ) is either ${ }_{j}^{O a}\left(q^{\prime}\left(x_{n}\right)-x_{j+1}\right)$ or 0 , depending on the value of $x_{n}$; here $q^{\prime}\left(x_{n}\right)$ is $q^{\prime}$ as defined in the proof of Lemma IA for $i=j$, $g\left(x_{j}\right)=h_{j}\left(x_{j}, x_{n}\right)$. The second term (corresponding to $T_{2}$ ) is $h_{j}\left(x_{j+1}+5, x_{n}\right) F_{j}\left(x_{j+1}+S\right)$, where s is the least upper bound of the set represented by $\mathrm{H}_{j}^{\mathrm{Oa}}(y)$.

Applying (52), we see that the contribution of the first term to (5I) cannot be greater than

$$
\int d x_{j+1} d x_{n} F_{n}\left(x_{n}\right) m_{j+2} \cdots m_{n-1} H_{j}^{0 a}\left(q^{\prime}\left(x_{n}\right)-x_{j+1}\right)=\left(k_{j}-a\right) \prod_{j+2}^{n} m_{n}(53)
$$

which approaches zero as $a \rightarrow k_{j}$. Therefore, in the case $A=k_{j}$, we can ignore this term in computing $g(A)$, and we have

$$
\begin{aligned}
g(A) & =\lim _{a \rightarrow A-} \int d x_{j+1} d x_{n} Q\left(a_{,} x_{j+1}, x_{n}\right) h_{j}\left(x_{j+1}+5, x_{n}\right) F_{1}\left(x_{j+1}+5\right) \\
& \left.=\prod_{i=1}^{j-\frac{\partial}{\partial a_{i}}}\right) J\left(a_{1} \ldots a_{j-1}\right)_{0}
\end{aligned}
$$


23.

in the notation of (18), where

$$
\begin{aligned}
& J\left(a_{1} \ldots a_{j-1}\right)=\int \pi_{1}^{j-2}\left[F_{1}^{a_{i}}\left(x_{i}\right) H_{i}^{a_{i} a_{i+1}}\left(x_{i}-x_{i+1} j d x_{i}\right]\right.
\end{aligned}
$$

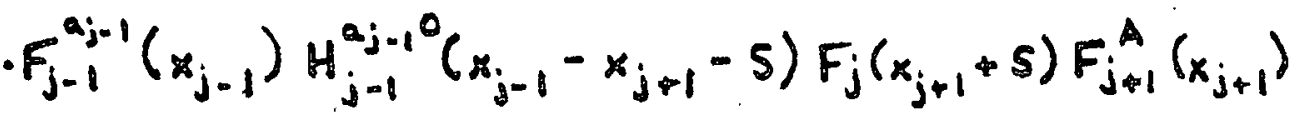

$$
\begin{aligned}
& H_{j+1}^{A 0}\left(x_{j+1}-x_{j+2}\right) d x_{j-1} d x_{j+1} \\
& \prod_{j+2}^{n-1}\left[F_{i}\left(x_{i}\right) H_{i}\left(x_{i}-x_{i+1}\right) d x_{i}\right] F_{n}\left(x_{n}\right) H_{n}^{O a_{1}}\left(x_{n}-x_{i}\right) d x_{n} \\
& g(A)=\int H_{n}\left(x_{n}-x_{2}-s\right) F_{1}\left(x_{2}+S\right) F_{2}^{A}\left(x_{2}\right) H_{2}^{A O}\left(x_{2}-x_{3}\right) d x_{2} \\
& \text { - } \prod_{3}^{n-1}\left[F_{i}\left(x_{i}\right) H_{i}\left(x_{i}-x_{i+1}\right) d x_{i}\right] F_{n}\left(x_{n}\right) d x_{n}
\end{aligned}
$$

In either (55) or (56) the product $F_{j}\left(x_{j+1}+S\right) F_{j+1}^{A}\left(x_{j+1}\right)$ is the characteristic function of a set of measure

$\leqslant \vec{m}=\min \left(m_{j}, m_{j+1}-A\right)$. Let us replace this product by $\bar{F}\left(x_{j+1}\right)$, the characteristic function of some set having measure $\bar{m}$ and including the original set. We may call the result of this replacement $\bar{g}(A)$; then (using the fact that $\left.h_{j}\left(x_{j+1}+S, x_{n}\right) \geqslant 0\right) \quad$ we have

$g(A) \leqslant \bar{g}(A)$

(57) 
24.

and also, from (17) and (26),

$$
m_{n} \leq \bar{m}
$$

We treat $* g(a)$ by applying Lemma II to eliminate the $\operatorname{variable} x_{j}$. In the graphical language of Note 2 , this leaves us (in the limit $a \rightarrow A=k_{j}$ ) with new lines $P_{j-1} P_{j+1}, P_{j+1} P_{0}, P_{0} P_{j-1}$ having "lengths" $k_{j-1}, m_{j}, k_{j-1}+m_{j}$. The third of these lines is made superfluous by the first two; since the condition $\left|x_{j-1}\right| \leq k_{2}\left(k_{j-1}+m_{j}\right)$ is implied by $\left|x_{j-1}-x_{j+1}\right| \leqslant \frac{1}{2} k_{j-1}$ and $\left|x_{j+1}\right| \leqslant \frac{1}{2} m_{j}$. Also the "longer" of the two lines now joining $P_{j+1}$ to $P_{0}$ may be dropped, leaving one line of "length" $\overrightarrow{\mathrm{m}}$ as defined above. (see Fig. 6.) With a suitable use of (13) for the variable $x_{j+1}$, we now have

$$
{ }^{*} g(A)=\prod_{1}^{j-1}\left(-\frac{\partial}{\partial a_{i}}\right) * J\left(a_{1} \ldots a_{j-1}\right)_{0} \quad \text { (59) }
$$

where

$$
\begin{aligned}
& * J\left(a_{1} \ldots a_{j-1}\right)=\int \pi_{1}^{j-2}\left[F_{i}^{* a_{i}}\left(x_{i}\right) H_{i}^{* a_{i} a_{i+1}}\left(x_{i}-x_{i+1}\right) d x_{i}\right] \\
& \text { - } F_{j-1}^{* a_{j-1}}\left(x_{j-1}\right) H_{j-1}^{* a_{j-1}}\left(x_{j-1}-x_{j+1}\right) \bar{F}_{j}^{*}\left(x_{j+1}\right) H_{j+1}^{A O *}\left(x_{j+1}-x_{j+2}\right) d x_{j-1} d x_{j+1} \\
& \prod_{j+2}^{n-1}\left[F_{i}^{*}\left(x_{i}\right) H_{i}^{*}\left(x_{i}-x_{i+1}\right) d x_{i}\right] F_{n}^{*}\left(x_{n}\right) H_{n}^{* O a_{1}}\left(x_{n}-x_{1}\right) d x_{n} \\
& \text { (6) }
\end{aligned}
$$


if $j>1$; and for $j=1$ we have

$$
\begin{aligned}
*_{g}(A)= & \int H_{n}^{*}\left(x_{n}-x_{2}\right) \bar{F}^{*}\left(x_{2}\right) H_{2}^{A O^{*}}\left(x_{2}-x_{3}\right) d x_{2} \\
& \prod_{3}^{n=1}\left[F_{i}^{*}\left(x_{i}\right) H_{i}^{*}\left(x_{i}-x_{i+1}\right) d x_{i}\right] F_{n}^{*}\left(x_{n}\right) d x_{n}
\end{aligned}
$$

where $\bar{F}^{*}$ is the characteristic function of $S_{\bar{m}}$. We may now apply Theorem IA with $\ell \rightarrow \ell, j \rightarrow j-l$,

$$
\begin{array}{r}
m_{j+1} \rightarrow \bar{m}, k_{j+1} \rightarrow k_{j+1}-k_{j}, \text { obtaining } \\
\bar{g}(A)=g(A) .
\end{array}
$$

Note that (16) is satisfied because by hypothesis $k_{j}<k_{j+1}$ and (17) is satisfied because of (58).

Combining (57) with (62), we have (28).

The proof of (37) when $B=k_{j}<\min \left(k_{j+1}, k_{n-1}\right)$ is quite similar, although some of the formulas are different. For example; in (55) the factors $H_{n-1}\left(x_{n-1}-x_{n}\right) F_{n}\left(x_{n}\right) H_{n}^{0,1}\left(x_{n}-x_{1}\right)$ are replaced by $H_{n-1}^{O B}\left(x_{n-1}-x_{n}\right) \sum_{n}^{B}\left(x_{n}\right) H_{n}^{B a}\left(x_{n}-x_{1}\right)$. of course, $a \rightarrow b$ and $A \rightarrow B$ throughout. The most important change is that we no longer have (58) but only

$$
m_{n}-k_{j} \leq \bar{m}
$$

This is sufficient to give (17) in the final application of Theorem IA with $j \rightarrow j-1$, as this application now 
involves the substitutions $m_{n} \rightarrow m_{n}-k_{j}, k_{n}-k_{j}, k_{n-1} \rightarrow k_{n-1}-k_{j}$ in addition to those previously listed.*

A snag arises if $k_{n}=k_{j}$ since (16) is not satisfied. This may be handled as follows. If $j=I$, the left side of (37) vanishes because both terms now have a form like (53). If $j>1$, we may eliminate both $x_{1}$ and $x_{j}$, reducing (37) to an application of Theorem IA with $j \rightarrow j-2$.

Note 4 .

By renumbering the indices in the order $j, \cdots, 1, n, \cdots, j+1$, we transform (35) into (34). The various $\mathrm{k}^{\prime} \mathrm{s}$ and $\mathrm{m}^{\prime} \mathrm{s}$ are thereby altered, but (17) is unaffected because of (29).

By the same transformation, the task of proving (37) when $B=k_{n}$ (Case $2 b$ ) is reduced to that of proving it when $\mathrm{B}=\mathrm{k}_{j}($ Case $2 \mathrm{a})$.

Note 5 .

If $\ell=2$, the indices $j+1$ and $n-1$ are identical, and therefore (30) involves a factor $H_{n-1}^{a b}\left(x_{n-1}-x_{n}\right)$. Similarly (31). Hence we must replace (33), still assuming that $\mathrm{j}>0$, by

$$
B=\min \left(\frac{1}{2} k_{n-1}, k_{j}, k_{n}, m_{n}\right) \quad(64)
$$

We observe that conditions (16) and (17) hold.if $m_{j+1} ; m_{n}, k_{j}, k_{n}$ are diminished by $b$ and $k_{n-l}$ is diminished by $2 b$, where 
$0 \leqslant b<B$. The proof of (36) now proceeds as before. In Case I of (37), we observe that both sides vanish if $B=\frac{1}{3} k_{n-1}$. The proof in the other cases is unaffected except for inessential changes in the formulas of Note 3 .

$$
\text { If } j=0,(30) \text { involves a factor } H_{n}^{b a}\left(x_{n}-x_{1}\right) \text {. }
$$

Therefore we replace (33) by

$$
B=\min \left(k_{1}, k_{n-1}, k_{2} k_{n}, m_{n}\right) \quad(6 s)
$$

if $l>2$, or by

$$
B=\min _{3}\left(\frac{1}{2} k_{1} \cdot k_{2} k_{2}, m_{2}\right) \quad(60)
$$

if $l=2$ (in this case $n=2$ ). The proof of $(36)$ is again unaltered. Both sides of (37) now vanish in all cases, so that Note 3 is not needed.

Appendix I.

It is possible to modify this proof so as to follow more closely the argument of [1] for $n=2$. In Theorem IA, one should replace (17) by

$$
m_{n} \equiv \min \left(m_{j}+\sum_{0}^{j-1} k_{i}, m_{j}+\sum_{j}^{n-1} k_{i}\right), j=1, \ldots, n-1
$$

which is equivalent to the assertion that the shortest path from $P_{0}$ to $P_{n}$, in the graphs of Fig. 5, is of length not less than $m_{n}$. Thus the conclusion of Note 2 - particularly: 
Eq. (21) - still holds good. we may now replace (26) by

$$
A=\min \left[k_{j+1}, k_{j}, \frac{1}{2}\left(m_{j+1}-m_{n}+\sum_{0}^{j} k_{i}\right), \frac{1}{i}\left(m_{j+1}-m_{n}+\sum_{i+1}^{n-1} k_{i}\right)\right]
$$

without disturbing the proof of (27). The proof of (28) is thus made easier, in that case 3 becomes

$$
A=\frac{1}{2}\left[m_{j+1}-m_{n}+\min \left(\sum_{0}^{j} k_{i} \cdot \sum_{j+1}^{n-1} k_{i}\right)\right]
$$

and (29) is replaced by

$$
m_{n}=m_{j+1}+\min \left(\sum_{0}^{j} k_{i} \cdot \sum_{j+1}^{n-1} k_{i}\right)
$$

To prove (25) in the presence of (70), we first replace $F_{n}\left(x_{n}\right)$ and $F_{n} *\left(x_{n}\right)$ by 1 for all $x_{n}$; this cannot increase $* g(0)$ because of $(70)$. Then we renumber the indices as in Note 4, and proceed as in the text following (25) except that we now are entitled to put

$$
A=\min \left(k_{j+1}, k_{j}\right)
$$

Since Case 3 is now ruled out, the proof is complete, except for details the reader may supply.

The advantage of this version is that it bypasses the "simultaneous truncation" leading to Eqs. (30)-(37). The price paid is the more elaborate form of (67) as opposed to (17). However, the difficulties dealt with in Note 3 are unchanged. 
29.

Appendix II.

An analogue of Theorem I for discrete sets may be stated as follows:

Theorem II

Let $m_{1}, \ldots, m_{n}, k_{1}, \ldots, k_{n}$ be non-negative integers, satisfying

$$
m_{i}+m_{i+1}+k_{i} \equiv 1(m c d 2)
$$

for $\leqslant i \leq n$. (As before we understand $m_{n+1}$ to mean $m_{i}$, etc.) For each $i=1, \ldots, n$ let $\phi_{i}$ be a set of $m_{i}$ odd (even) integers, $m$ being even (odd), and let $\psi_{i}$ be a set of $k_{i}$ odd (even) integer $s_{,} k_{i}$ being even (odd). Let $\phi_{i}^{*}$ be the set $\left\{m_{i}-1, m_{i}-3, \ldots,-m_{i}+1\right\}$, and let. $y_{i}^{*}$ be the set $\left\{k_{i}-1, k_{i}-3, \ldots,-k_{i}+1\right\}$. Let I be the number of n-tuplets $\left\{x_{1}, \ldots, x_{n}\right\}$ satisfying

$x_{i} \varepsilon \phi_{i}, x_{i} x_{i+1} \varepsilon \psi_{i}$

$(73)$

for each $i$; and let I be the number of n-tuplets satisfying

$$
x_{i} \varepsilon \phi_{i}^{*}, x_{i}-x_{i+1} \varepsilon \psi_{i}^{*} \quad(74)
$$

for each i. Then

$$
I \equiv{ }^{*} I
$$

(75) 
(The restriction to odd or even integers is a device to allow symmetrization of both even and odd sets. Condition (72) is needed to make the sets match. Doing without this condition would entail complications we prefer not to discuss.)

We sketch a proof of this theorem, parallel to the one given in the text for Theorem $I$. First, an operation of truncation must be defined, by which, for any non-negative integer a and any set $\phi$ of at least a integers, the set $\phi^{a}$ is obtained by deleting the top a members of $\phi$; and for non-negative $a, b$ and set $\psi$ of at least $a+b$ integers, $\psi^{a b}$ is obtained by deleting the top $a$ and bottom $b$ members of $\psi$. This leads to definitions of $I\left(a_{1}, \ldots, a_{n}\right)$ and $* I\left(a_{1}, \ldots, a_{n}\right)$ like (14) and (15). Then we define the operator $\Delta_{c}$ such that for any function $f(a)$ we have

$$
\Delta_{a}+\left(r_{k}\right)=f(a)-(a+1) .
$$

We can now state the analogue to Eqs. (16)-(18):

Theorem IIA

Suppose' that

$$
\begin{aligned}
& k_{i}>0 \quad \text { fir } i=j \text { or } n \\
& k_{i}=1 \quad \text { fer } i=1, \ldots, j-1 \\
& 1 \leq m_{n} \leq m_{i} \text { for } i=1, \cdots, n-1 .(7)
\end{aligned}
$$

Then

$$
\prod_{1}^{j} \Delta_{a_{i}} I\left(a_{1}, \ldots, a_{n}\right) \leq \prod_{0}^{j} \Delta_{a_{i}}^{*} I\left(a_{1}, \ldots, a_{n}\right)
$$


(Note that $(77)$ and (78) are required to ensure that the operations in (80) are defined.)

We shall merely indicate how the proof of this theorem differs from that of Theorem IA.

The reasoning of Note 1 is unimpaired. The sets $T_{1}$ and $\mathrm{T}_{2}$ do not overlap at all.

The reasoning of Note 2 is unchanged except that in

Lemma IIA we obtain $p_{1}+p_{2}-1, p_{i}+k_{i}-1, p_{2}+k_{i}-1$

instead of $p_{1}+p_{2} \cdot p_{1}+k_{i} \cdot p_{2}+k_{i}$. Therefore, to maintain the invariance of "shortest path length" under the graphical operations, we must take the "length" of a line to be 1 less than the number of elements in the corresponding set. Conditions (77)-(79) prevent the occurrence of negative "lengths", since $j \equiv n-\ell=n-1$ in this part of the proof.

Equation (27) is replaced by

$$
\Delta_{a} g(a) \leq \Delta_{a} \cdot g(a)
$$

which follows by induction on $\ell$ provided that a is small enough to satisfy

$$
k_{j+1}-a>0, k_{j}-a>1, m_{j+1}-a \geqslant m_{n} \cdot(82)
$$

But we need only prove (81) for a A; therefore, instead of (26), we have

$$
A=\min \left(k_{j+1}, k_{j}-1, m_{j+1}-m_{n}+1\right) \cdot(B j)
$$


32.

This leads, as before, to three cases in the proof of (28).

In Case $1\left(A=k_{j+1}\right)$ both sides of (28) vanish.

In Case $2\left(A=k_{j}-1\right)$ the operator $\Delta_{a_{j}}$ can be aropped from both sides of (28), since the set $\psi^{\text {lA }}$ is empty. Then, since $\psi_{i}^{O A}$ has only one member, the variable $x_{j}$ can be eliminated and we arrive at formulas like (55) and (59) of Note 3 . The arguments of Note 3 are otherwise unchanged. In Case $3\left(A=m_{i+1}-m_{n}+1\right)$ we are faced with the problem of proving (25) with the condition

$$
m_{j+1}=m_{n}-1
$$

which violates (79). Actually, this condition makes the proof easier.. We introduce an additional induction on $m_{n}$. If $m_{n}=1$, the proof is completed for case 3 by noting that (84) makes both sides of (25) vanish. If $m_{n}>1$, we treat Case 3 by renumbering the indices according to Note 4 ; condition (79) is thus restored and (25) becomes an instance of the theorem with $m_{n} \rightarrow m_{n}-1$. Note that $m_{n}$ remains fixed throughout the double induction on and $j$ elsewhere in the proof.

The simultaneous truncation described in Eqs. (30)(37) is thus bypassed in the discrete version of the theorem without the need to introduce the more complicated condition (67) 
By setting $j=0$ in Theorem IIA, we obtain Theorem II except for the case where some $m_{i}$ or $k_{i}$ vanishes; but in this case we have $T=T=0$.

Appendix III.

we conjecture the following generalization of (8).

Let $\{$ ir $\}$ be an array of real numbers, where

$$
\begin{aligned}
& i=1, \ldots, n \\
& \gamma=1, \ldots, r
\end{aligned}
$$

Let $H_{1}, \ldots, H_{p}$ be functions from the interval $(-\infty, \infty)$ to the interval $[0, \infty)$. Let $H_{0}^{*}, \ldots, H_{r}$ be their symmetric decreasing rearrangements. Then

$$
\left.\int \prod_{i}\left(d x_{i}\right) \pi_{\gamma} H_{\gamma}\left(\sum_{i} u_{i \gamma} \dot{x}_{i}\right) \geq \int \pi_{i}\left(d x_{i}\right) \prod_{\gamma} H_{\gamma}^{*}\left(\sum_{i} u_{i \gamma} x_{i}\right) .88 .7\right)
$$

To obtain Theorem I, set $r=2 n, u_{i j}=\delta_{i y}+\delta_{i, \gamma-n}-\delta_{i-1, \gamma-n}-\delta_{i,} \delta_{\gamma}$. We have been unable to prove even the following two special cases of (87):

$$
\begin{aligned}
& \int \prod_{i}\left[H_{i}\left(x_{i}-x_{i+1}\right) H_{i+n}\left(x_{i}+x_{i+1}\right) d x_{i}\right] \\
& \leq \int \prod_{i}\left[H_{i}^{*}\left(x_{i}-x_{i+1}\right) H_{i+n}\left(x_{i}+x_{i+1}\right) d x_{i}\right]
\end{aligned}
$$


and

$$
\int \prod_{i=1}^{n-1}\left[d x_{i} \prod_{j=i+1}^{n}+t_{i j}\left(x_{i}-x_{j}\right)\right]=\int \prod_{i=1}^{n-1}\left[d x_{i} \prod_{j=i+1}^{n} \|_{i j}^{*}\left(x_{i}-x_{j}\right) \mid+(\infty)\right.
$$

The difficulty arises in Lemma IB, which breaks

down when the same function is truncated twice from the same end. 


\section{FIGURE CAPTIONS}

Figure 1. Examples of truncation.

Each example depicts a subset of the real line. If $F$ is the characteristic function of the set $\left[r_{1}, r_{2}\right] \cup\left[r^{\prime}, r_{4}\right] \cup\left[r_{5}, r_{6}\right]$ depicted by $(a)$, then $F^{a}$ is the characteristic function of the set depicted by (b), if a $a r_{6}^{-r} 5^{\prime}$ or by (c), if $r_{6}^{-r} 5<a<r_{6}^{-r} 5+r_{4}^{-r} 3$. Likewise, if $H$ corresponds to (d), then $H^{a b}$ corresponds to (e) if $b>r_{2}^{-r}$ and $a+b<r_{4}^{-r_{3}}+$ $r_{2}{ }^{-r}{ }_{1}$. Note that a affects only the "top" of example (e) and (b) only' the "bottom":

Figure 2. Truncation and rearrangement.

Examples (a), (b), (c), (d), (e) depict the functions $F, F^{a}, F^{*}, F^{*}$, and $F^{a^{*}}$ respectively. Note that (d) and (e) are related by Eq. (13).

Figure 3. Case 1 of Lemmo $1 B$.

Examples $(a)-(e)$ depict $g\left(x_{i}\right), F_{i}\left(x_{i}\right), g\left(x_{i}\right) F_{i}\left(x_{i}\right), H_{i}(y)$ and $G\left(x_{i}+i\right)$. Here $q_{1}>q_{2}$ and hence. $q^{r}=q_{2}$. Since $g\left(q^{\prime}\right)=0$, a simultaneous infinitesimal truncation of (a) and (b) from above will have no effect on (c). Therefore $G$ vanishes unless the point $x_{i+i}+q_{3}$ lies in (c). Thus (e) is the same as (c), displaced by $q_{3}$. Clearly (e) is unaffected by a truncation of (d) from below, since $q_{3}$ is unchanged.

Figure 4. Case 2 of Lemma IB.

Same five examples as in Figure 3. Here $g\left(q^{\prime}\right)=1$, so that a truncation of (a) and (b) also truncates (c). Therefore (e) consists of $T_{l^{\prime}}$ for which 
$q^{\prime}=x_{i+i}$ lies in (d), as well as $T_{2}$, for which $x_{i+i}+q_{3}$ lies in (c). Truncation of (d) at $y=t$ results in truncation of (e) at. $x_{i}+1=$ $q^{\prime}-t$.

Figure 5. The first five graphs described in Note 2.

Example (a) shows the original graph (points $P_{4}$ to $P_{n-1}$. not shown). In (b), the variable $x$, has been eliminated by the use of Lemma II. In (c), the superfluous line $P_{0} P_{2}$ has been dropped. (We write $\bar{m}_{2}$ for $\min$ $\left.\left(m_{2}, m_{1}+k_{1}\right)_{0}\right)$ In (d) we have eliminated $x_{2}$, and in (e) we have again dropped superfluous lines, putting $\bar{m}_{3}=\min \left(m_{3^{\prime}} \bar{m}_{2}+k_{2}\right)$, $\bar{m}_{n}=\min \left(k_{n}+m_{1}>k_{n}+k_{1}+\bar{m}_{2}\right)$. Note that if any graph contains a path from $P_{0}$ to $P_{n}$ with total length $L$, then all the other graphs contain some such path with length $L \leq L$.

Figure 6. Graphical treatment of the right side of (28) according to Note 3.

The original graph for " $g(a)$ is shown in (a). In (b) we have used Lemma II to eliminate the variable $x_{i}$. In (c) we have replacea a by $A=k_{i}$. In (d) we have dropped superfluous lines; $\bar{m}=\min \left(m_{i}, m_{i}+i-A\right)$. 


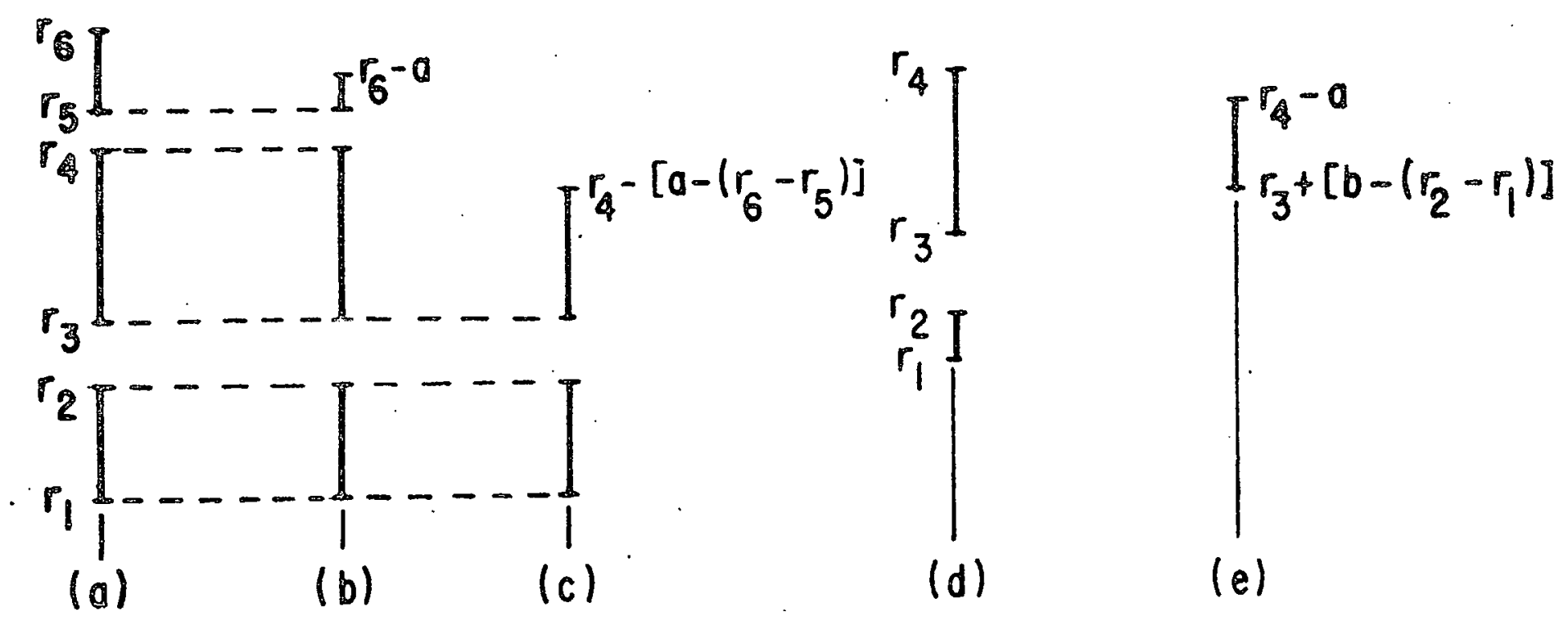




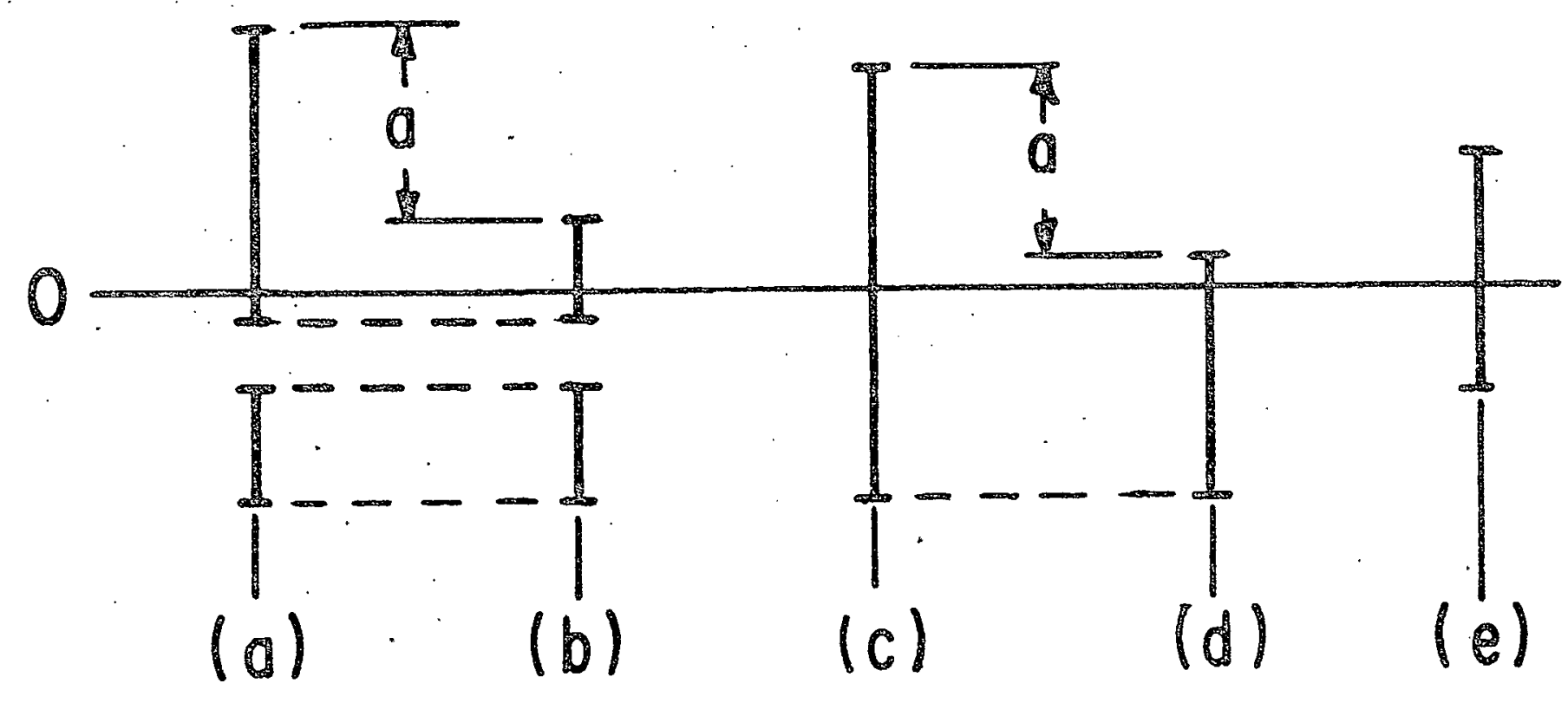




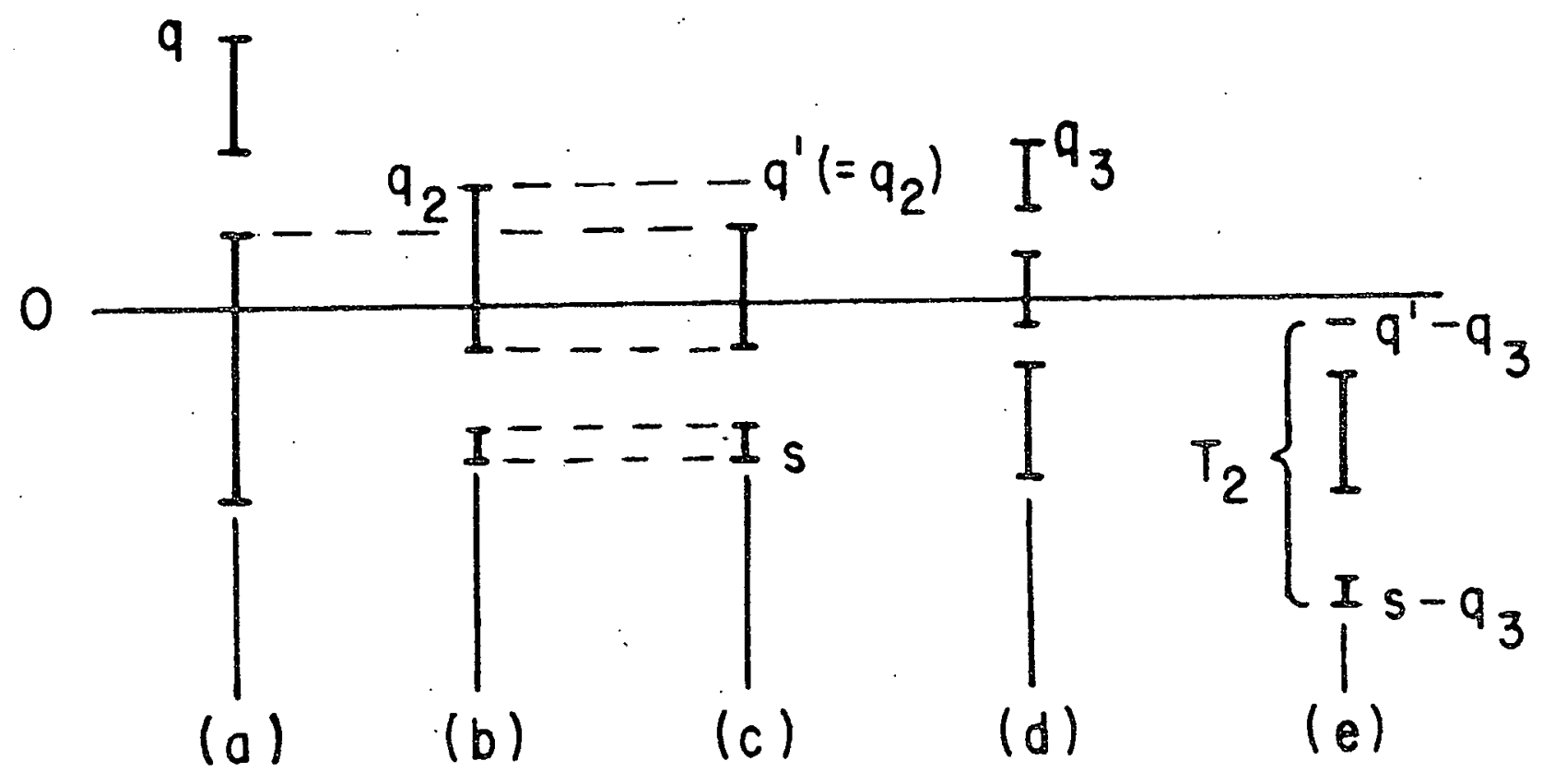




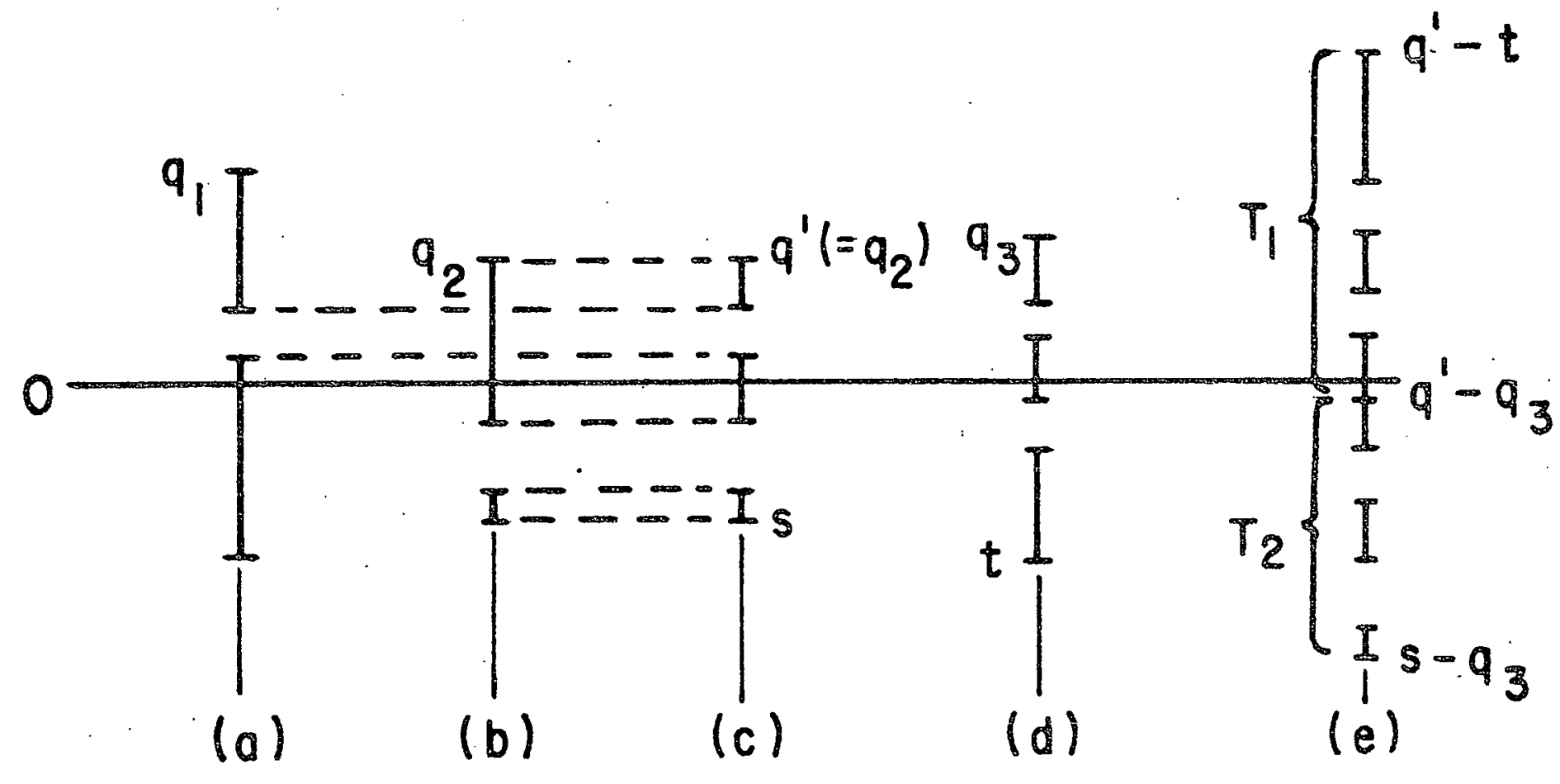




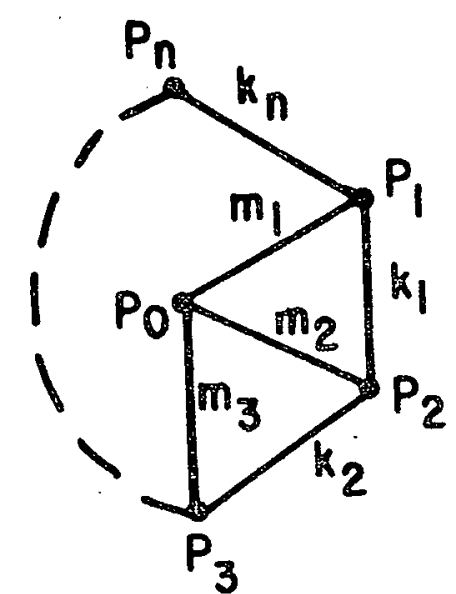

(a)

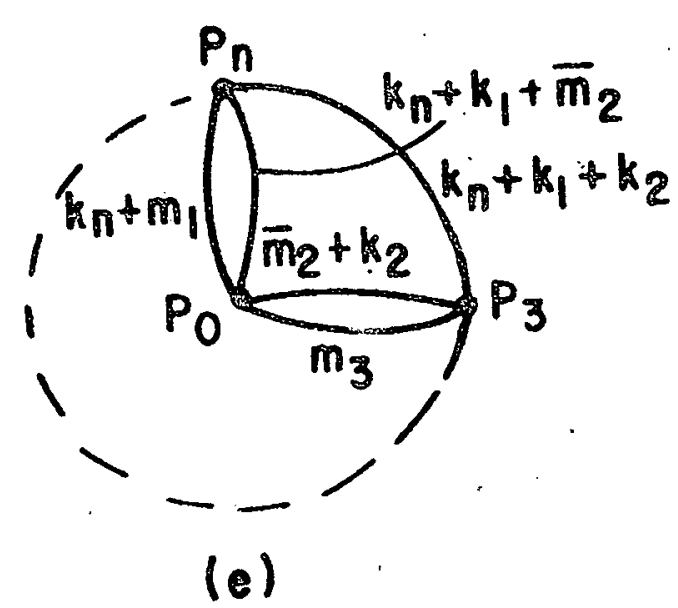

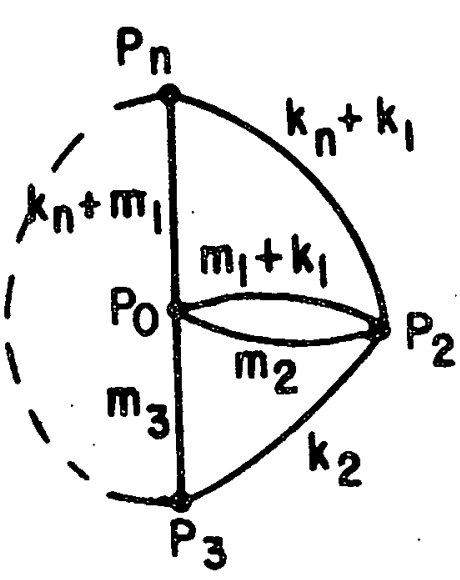

(b)

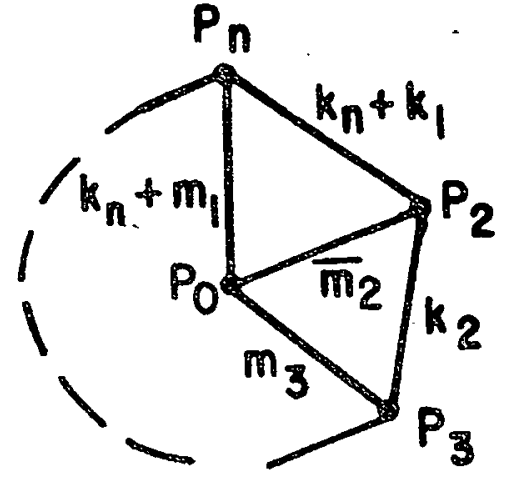

(c)

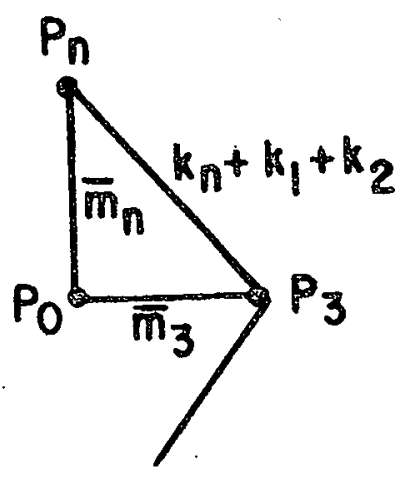

(f) 


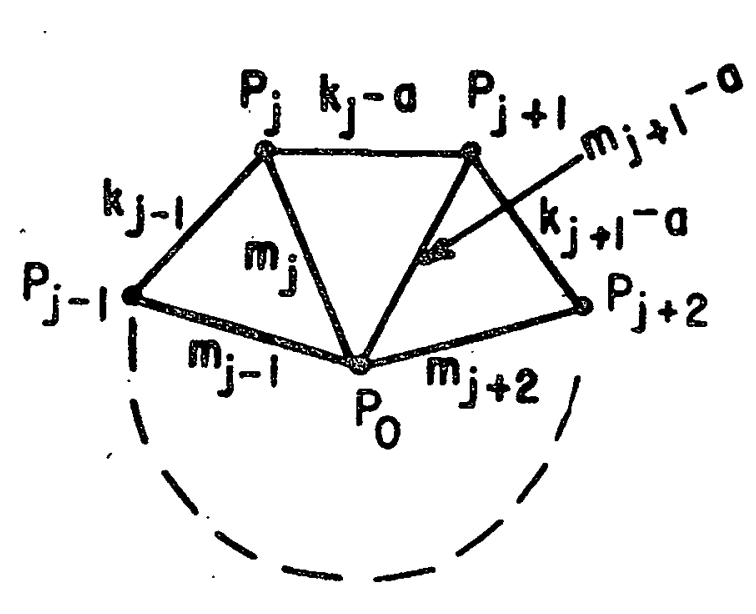

(a)

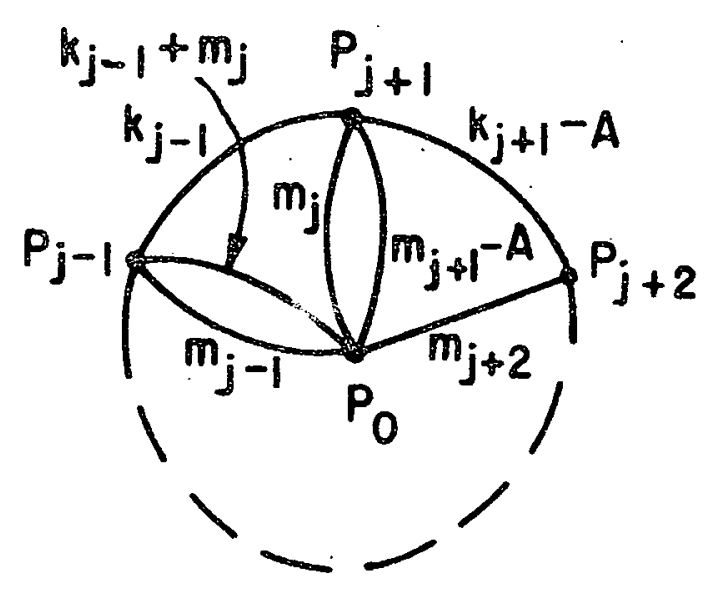

(c)

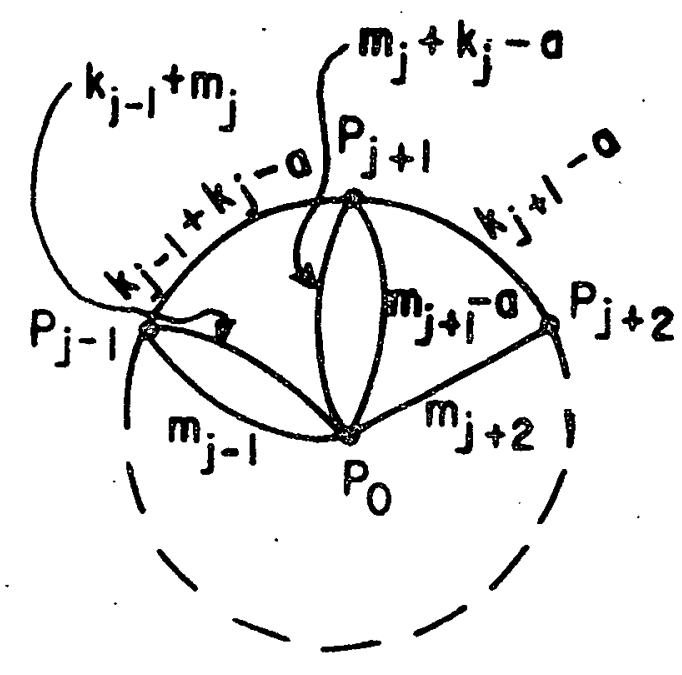

(b)

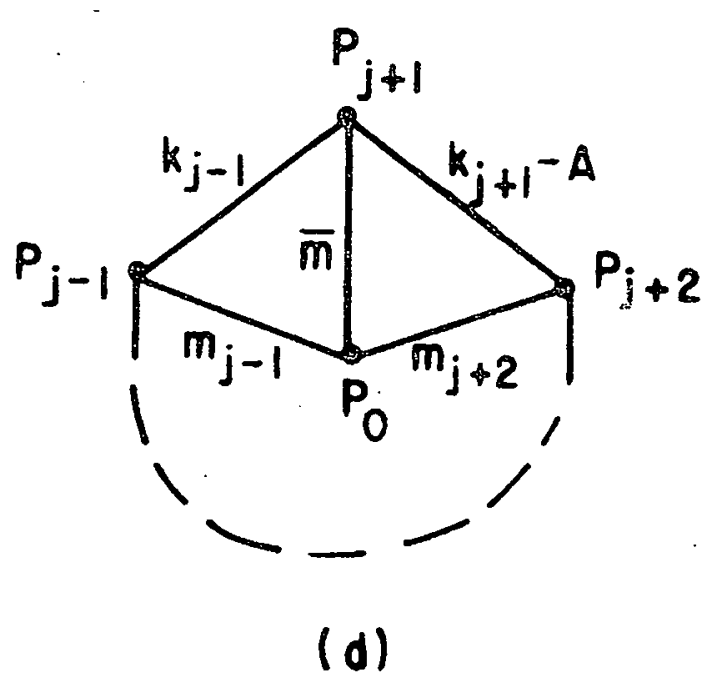

